\title{
Presentación inhabitual de un caso de neumonía bulosa aguda bilateral por Pneumocystis jiroveci complicada con neumotórax
}

\author{
Rolando Pizarro P., Claudio Valdés H. y Jaime Vitali C.
}

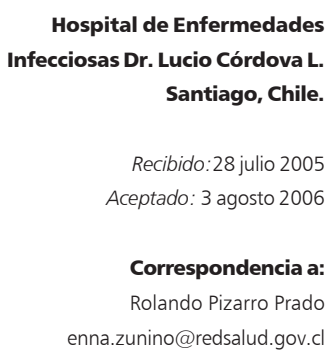

\section{Unusual presentation of bilateral bullous Pneumocystis jiroveci pneumonia complicated with pneumothorax}

In patients with human immunodeficiency virus infection, pneumothorax overcomes in advanced stages of the illness, associated with infections by Mycobacterium tuberculosis or Pneumocystis jiroveci, in smokers and intravenous drug users. We present a case with this unusual complication: homosexual man, 30 years of age, with a history of one month of cough, progressive dyspnea, weight loss and diarrhea. He was hospitalized with the diagnosis of atypical pneumonia, respiratory failure and a presumptive HIV infection. His clinical course was complicated by the presence of bullae and pneumothorax by day 15 of hospitalization. The existence of Pneumocystis jiroveci in sputum was confirmed by a direct immunofluorescence test. The patient was treated with sulpha-trimethoprim, steroids, oxygen, but he died 21 days after admittance. The strategies recommended for treatment of pneumothorax appeared during $P$. jiroveci pneumonia in HIV positive patients are summarized.

Key words: Pneumothorax, bullous pneumonia, Pneumocystis jiroveci.

Palabras claves: Neumotórax, neumonía bulosa, Pneumocystis jiroveci.

\section{Introducción}

$\mathrm{E}$ n los pacientes infectados por el virus de la inmunodeficiencia humana (VIH), en etapas avanzadas de esta enfermedad, el neumotórax se observa con cierta frecuencia y es un evento de mal pronóstico, en cambio la enfermedad bulosa pulmonar no está descrita en forma relevante.

Nos pareció de alto interés clínico comunicar un caso cuya forma de presentación fue de inicio brusco, con enfermedad bulosa pulmonar y neumotórax, causada por Pneumocystis jiroveci en un paciente con infección por VIH, no diagnosticada previamente a su ingreso.

\section{Caso clínico}

Varón de 30 años de edad. Refería tos seca con una data de un mes, sudoración nocturna, dificultad respiratoria progresiva y baja de peso estimada en $10 \mathrm{~kg}$. No refería fiebre y una semana antes de ser internado se le agregó diarrea líquida. Aportó el antecedente de promiscuidad homosexual y gonorrea 8 años antes. Ingre- só vigil, orientado, temperatura de $37^{\circ} \mathrm{C}$, presión arterial de $100 / 65$, pulso de 108 /minuto, taquipneico con frecuencia respiratoria de 50/minuto. Hechos destacables al examen físico de ingreso eran: adenopatías pequeñas, móviles, no adheridas a planos profundos e indoloras, tanto cervicales laterales y posteriores así como axilares e inguinales; boca con placas blanquecinas que comprometían la lengua, cara interna de mejillas y faringe; pulmones con estertores finos en ambas bases. El examen cardiovascular y abdominal carecía de hallazgos patológicos. Se plantearon las hipótesis diagnósticas de: neumopatía atípica; insuficiencia respiratoria; candidiasis orofaríngea; ¿infección por VIH? Se inició terapia con cotrimoxazol oral $1.600 \mathrm{mg}$ (sulfa) cada 8 horas e itraconazol $100 \mathrm{mg}$ cada 12 horas, más prednisona $40 \mathrm{mg}$ al día y oxígeno al $50 \%$.

En la exploración de laboratorio tenía: LDH de 833 $\mathrm{U} / \mathrm{L}$; hematocrito $44 \%$; leucocitos $6.900 \mathrm{x} \mathrm{mm}^{3}$ (segmentados $87 \%$, linfoncitos $8 \%$ ). Radiografía de tórax con infiltrados bilaterales, sin otras alteraciones (Figura 1).

Evolucionó mal, con taquipnea y polipnea, manteniendo aporte de $\mathrm{FiO}_{2}$ al $50 \%$ para alcanzar saturaciones de $90-92 \%$, siendo trasladado el quinto día a la Unidad de Tratamiento Intermedio Infectológico del Hospital, 


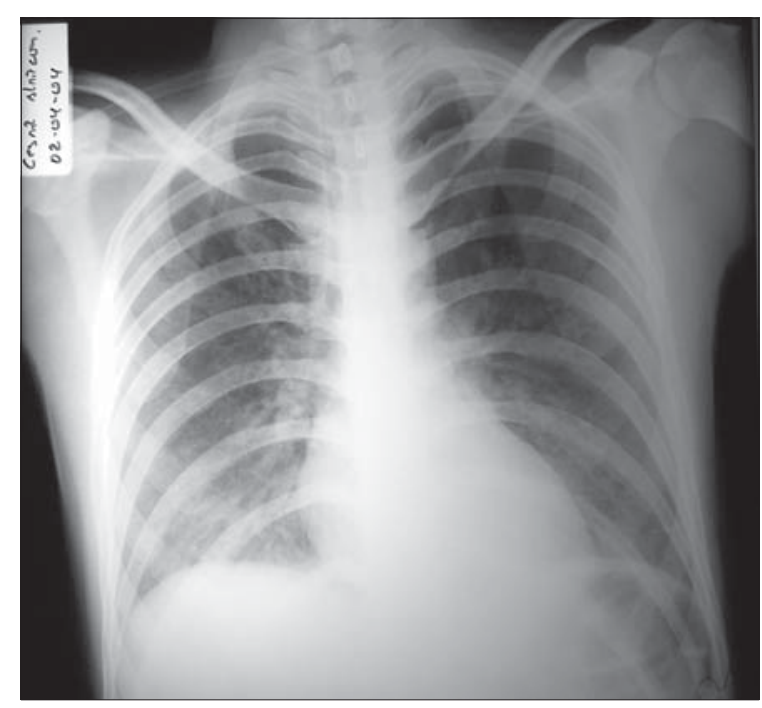

Figura 1. Radiografía de tórax de ingreso. Se observa abundante infiltrado intersticial bilateral.

donde se agregó a la terapia antimicrobiana ceftriaxona ev $1 \mathrm{~g}$ cada $12 \mathrm{~h}$ y claritomicina oral $500 \mathrm{mg}$ cada $12 \mathrm{~h}$.

A los 15 días de su ingreso presentó dolor retroesternal intenso. Una nueva radiografía de tórax evidenció múltiples lesiones de aspecto buloso, así como neumotórax en ambos campos pulmonares (Figura 2). Evaluado en conjunto por especialistas broncopulmonares, cirujanos de tórax e infectólogos, se hizo el diagnóstico de enfermedad bulosa bilateral con neumotórax menor al $15 \%$, lo que desaconseja el uso de tubo pleural. Además, se planteó la contraindicación de

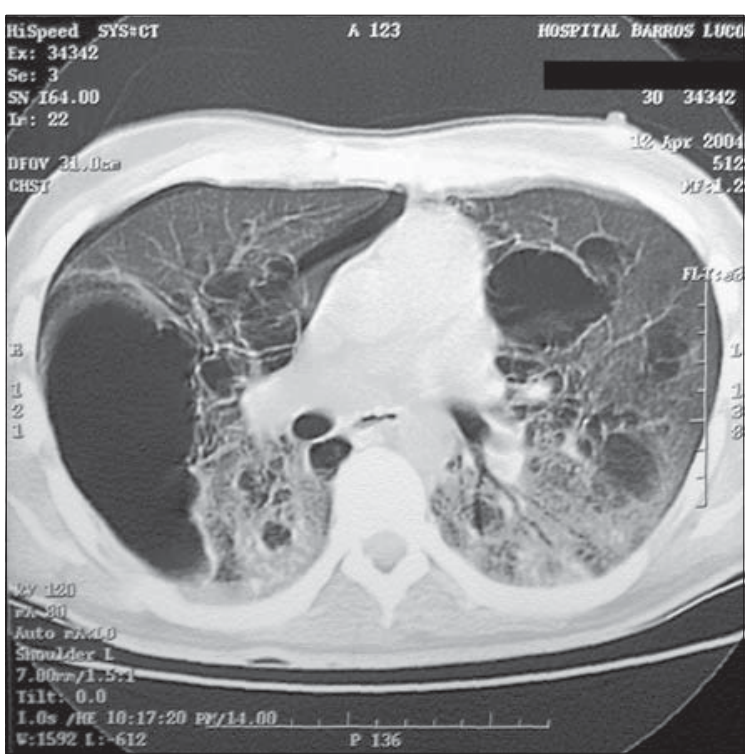

Figura 3 . TAC de tórax confirma la presencia de bulas gigantes bilaterales y enfisema pulmonar.

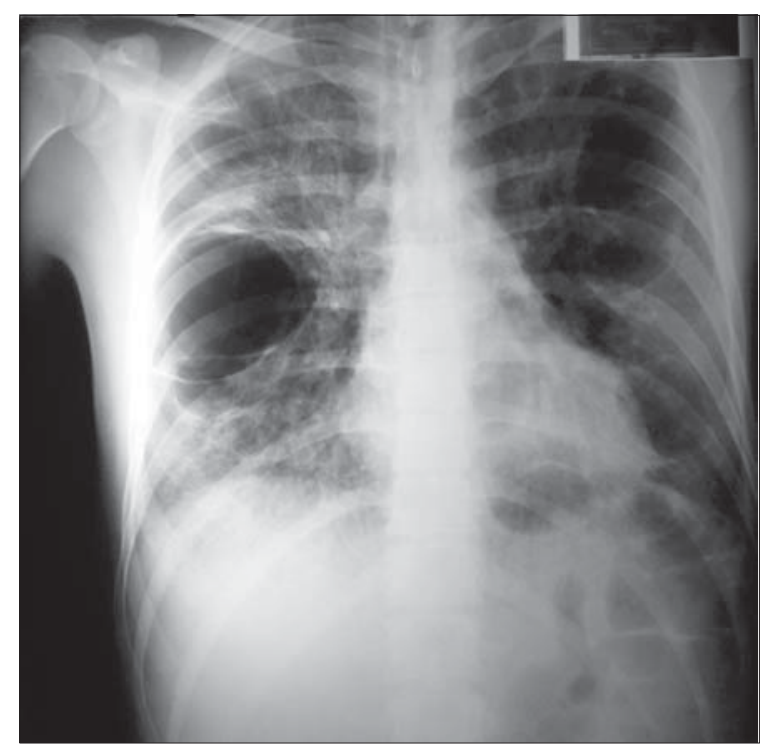

Figura 2. Radiografía de tórax 15 días post ingreso. Evidencia múltiple lesiones de aspecto buloso y neumotórax en ambos campos pulmonares. efectuar ventilación mecánica. Una TAC de tórax confirmó este diagnóstico (Figura 3). El test de ELISA para VIH fue $(+)$ y con posterioridad confirmado en el laboratorio de referencia (ISP). Se documentó la presencia de $P$. jiroveci por test de IFD. El recuento de linfocitos CD4 fue $3 \mathrm{x} \mathrm{mm}^{3}$.

Ante la pobre respuesta el día $17^{\circ}$ de su hospitalización, se adicionó a la terapia mencionada vancomicina, amikacina y terapia antituberculosa con isoniacida, rifampicina, pirazinamida y etambutol. Los requerimientos de oxígeno se elevaron, quedando con mascarilla de alto flujo. Las radiografías posteriores mostraron una rápida progresión del tamaño de las bulas ya existentes y la aparición de nuevas imágenes en ambos hemitórax. El equipo de cirugía de tórax reevaluó el caso, aconsejando una conducta expectante, pues la colocación de un tubo intercostal o la cirugía abierta empeorarían la situación del paciente, además que tenía pocas posibilidades de tolerarlo. La colocación de una válvula de Heimlich sería una indicación óptima, pero sólo con un aumento del neumotórax. Se mantuvo estable en su gravedad hasta el día $21^{\circ}$ de su hospitalización en que nuevamente comenzó con intenso dolor toráxico derecho, gran apremio ventilatorio, cianosis, aumentando la frecuencia respiratoria sobre $50 / \mathrm{min}$. Se le practicó una punción toráxica con fines evacuadores, sin lograrse ningún resultado, falleciendo el paciente a menos de una hora de iniciada esta crisis.

\section{Discusión}

Pneumocystis jiroveci es un patógeno relacionado frecuentemente, aunque no en forma exclusiva, con 
etapas avanzadas de la infección por VIH. Generalmente, provoca neumonías graves en pacientes con linfocitos CD4 bajo 200 células/ $\mathrm{mm}^{3}$ con predominio sintomático de tos, fiebre y disnea. La radiología muestra mayoritariamente infiltrados de aspecto retículo nodular, pero también puede existir consolidación o ser normal. La terapia de elección es cotrimoxazol, y una segunda alternativa es pentamidina endovenosa. Evoluciona en general en forma satisfactoria, pero algunos casos presentan complicaciones que hacen necesario el apoyo ventilatorio invasor en unidades de cuidados intensivos y se asocian a un incremento de la letalidad ${ }^{1,2}$.

En los pacientes con infección por VIH la ocurrencia del neumotórax es infrecuente, aunque se ha observado un incremento los últimos años, teniendo una relación inversamente proporcional al número de linfocitos CD4, con frecuencia que varía entre $2,7 \%$ y $4,9 \%$, mucho mayor que en pacientes no inmunocomprometidos $^{3,4}$. Dentro de los pacientes con infección por VIH esta incidencia puede llegar a $9 \%$ si coexiste infección por $P$. jiroveci; en los no co-infectados la frecuencia es virtualmente nula ${ }^{5}$. Se ha reportado su presentación bilateral hasta en $34 \%$ de los $\operatorname{casos}^{6,7}$.

La aparición de neumotórax está relacionada en forma creciente al uso de drogas intravenosas, con invasión del espacio pleural por inyecciones cervicales y drogas inhalatorias, que actúan como factores obstructivos; existe también una relación directa con el hábito tabáquico ${ }^{7,8}$. El uso de ventilación mecánica es otra causa que se relaciona con aparición de neumotórax, en este caso iatrogénica, así como la colocación de vías centrales y la toracocentesis ${ }^{9,10}$. El trauma también se menciona como causa de esta complicación. La terapia corticoesteroidal también aumenta la frecuencia del neumotórax y la morbilidad asociada, observándose una prolongación de los tiempos de uso de los tubos pleurales y un aumento de las terapias con pleurodesis química y quirúrgica. Esto se relaciona a la inhibición de la proliferación fibroblástica y de la síntesis proteica inducida por este grupo de fármacos ${ }^{6}$.

Entre las causas infecciosas de neumotórax se han identificado como relacionados los siguientes factores: la tuberculosis, las infecciones por mycobacterias atípicas, las neumonías bacterianas y la rotura de infartos pulmonares sépticos ${ }^{7,10,11}$. Pero dentro del contexto del neumotórax espontáneo, la infección pulmonar por $P$. jiroveci es innegablemente la causa más importante, originándose en la rotura de una o varias cavidades pulmonares subpleurales asociadas a necrosis de tales zonas, con la consiguiente formación de ampollas o bulas ${ }^{5,8,10-12}$. Algunas publicaciones describen una asociación entre las infecciones respiratorias a repetición en pacientes con infección por VIH, y la aparición de neumotórax, así como un incremento del riesgo al usar pentamidina aerosolizada como profilaxis para la neumonía por $P$. jiroveci $i^{7,11,12}$.

Los pacientes infectados por VIH que llegan a desarrollar neumotórax tienen un pronóstico sombrío. El impacto de la aparición de neumotórax se refleja en incremento de su estadía media a 9 días frente a 5 días en los pacientes sin neumotórax; traslados a una Unidad de Cuidados Intensivos con mucha mayor frecuencia (54 versus $11 \%$ ) y aumento de la mortalidad a $30 \%$ versus $5 \%$ en los pacientes que no presentan neumotórax ${ }^{10}$.

Las terapias indicadas ante la aparición de neumotórax en un paciente con infección por VIH son variadas pero con pobre respuesta, especialmente las causadas por infecciones, pues muchas cursan con fístula broncopleural prolongada, a consecuencia de las alteraciones del parénquima subyacente ${ }^{7}$. La utilización de tubos intercostales conectados a un sistema cerrado subacuático con aspiración continua y presiones reguladas es la primera técnica quirúrgica usada en estos casos y la de mayor frecuencia, con buenos resultados en neumotórax pequeños, unilaterales y sin fístula broncopleural ${ }^{4,7,10}$. Si falla la reexpansión pulmonar en un período de al menos 48 horas o el neumotórax se complica, el tubo tiende a fracasar y se debe pasar a otras terapias más avanzadas ${ }^{13,14}$, ya sea en pacientes seropositivos o negativos para VIH. La válvula de Heimlich, que permite la salida del aire subpleural, abriéndose con presión pleural positiva, ha demostrado su utilidad en aquellos individuos con neumotórax persistente por fistulisación. Además es segura y de fácil manejo ambulatorio, lo que permite el alta precoz, aunque se describe como su principal riesgo la colonización por microorganismos piogénicos, permitiendo posteriormente el desarrollo de empie$\operatorname{mas}^{7,15,16}$

Fracasadas las técnicas mencionadas, y siempre que las condiciones ventilatorias y hemodinámicas del paciente lo permitan, frente a la falla en la reexpansión pulmonar, el siguiente paso es la esclerosis con fibrina, bleomicina, talco o doxiciclina, sin éxito uniforme aún con procedimientos repetidos y utilizando videotoracoscopia $^{15-22}$. Si aún así no se obtienen resultados favorables, hay que considerar el engrapamiento toracoscópico o la toracotomía, pero llegar a estas instancias deteriora más el pronóstico de por sí malo en un paciente con estas características ${ }^{15-18}$.

El caso analizado, un paciente portador de infección por VIH, con infección intercurrente por $P$. jiroveci, presentó ciertas particularidades. Primero debutó en forma bastante agresiva, como una neumopatía aguda en un paciente con desconocimiento previo de la con- 
dición de seropositividad para VIH; luego, desarrolló en cosa de pocos días una enfermedad bulosa pulmonar, con cierto grado de neumotórax, estabilizado al principio con terapia acorde a la etiología causante de la complicación, pero rápidamente se apreciaron significativos cambios radiológicos. Esta misma situación desaconsejó recurrir a una solución quirúrgica, salvo que se produjera un incremento del neumotórax. Se amplió el esquema terapéutico, aunque no se planteó un fracaso de la terapia con cotrimoxazol; tampoco se inició terapia anti-retroviral. La crisis final fue tan brusca que impidió efectuar alguna maniobra de utilidad como intentar poner una válvula de Heimlich o un tubo intercostal. Se puncionó el lado afectado, pero el paciente falleció rápidamente, como se describe en estas circunstancias.

Es la primera vez que se presenta un caso con las características recién descritas en nuestra institución, a pesar de ser un centro de referencia para pacientes infectados por VIH, y en la literatura médica esta forma de evolución de la neumonía por $P$. jiroveci aparece descrita con baja frecuencia. Nos pareció interesante dar a conocer esta forma evolutiva como una manifestación poco habitual de esta enfermedad.

\section{Resumen}

En los pacientes con infección por el virus de la inmunodeficiencia humana (VIH), el neumotórax se observa generalmente en etapas avanzadas de la enfermedad, asociado a patógenos como Mycobacterium tuberculosis o Pneumocystis jiroveci, en individuos tabáquicos y drogadictos endovenosos. Presentamos un caso de presentación inhabitual: varón homosexual, 30 años de edad, con tos durante un mes, dificultad respiratoria progresiva y baja de peso, agregándose posteriormente diarrea. Fue hospitalizado con los diagnósticos de insuficiencia respiratoria, neumopatía atípica y observación de infección por VIH. Evolucionó mal, complicándose a los 15 días con neumotórax y bulas pulmonares. El LDH elevado y después la inmunofluorescencia directa $(+)$ confirmaron la etiología: $P$. jiroveci. El test de ELISA confirmó la pre-existencia de una infección por VIH. A pesar de una terapia enérgica con cotrimoxazol, corticoesteroides y oxígeno, el paciente falleció a los 21 días de su ingreso. Se describen las estrategias recomendadas para el manejo del neumotórax que acontece durante la neumonía por $P$. jiroveci en los pacientes portadores de infección por VIH.

\section{Referencias}

1.- Pérez C, Almendárez C, Oddó D, Sirhan M, Labarca J, Acuña G, et al. Neumonía por Pneumocystis carinii en inmunodeprimidos: cuadro clínico, tratamiento y pronóstico. Rev Méd Chile 1994; 122: 154-8.

2.- Pérez C, Muller C, Castillo Y, Mella S, Acuña G, Labarca J, et al. Neumonía por Pneumocystis carinii: Factores predisponentes, manifestaciones clínicas y respuesta a tratamiento. Rev Chil Infect 1999; 16: 299-304.

3.- Bagheri K, Truitt T, Safirstein B H. Spontaneous pneumothorax in patients with acquired immunodeficiency syndrome (AIDS) [abstract]. Chest 1993; 103: 226S.

4.- Wait M A, Dal Nogare A R. Treatment of AIDS- related pneumothorax : a decade of experience. Chest 1994; 106: 693-6.

5.- Macclellan M D, Miller S B, Parsons P E, Cohn D L. Pneumothorax with Pneumocystis carinii pneumonia in AIDS: incidence and clinical characteristics. Chest 1991; 100: 1224-8.

6.- Metersky M L, Colt H G, Olson L K, Shanks T G. AIDS-related spontaneous pneumothorax: risk factors and treatment. Chest 1995; 108: 946-51.

7.- Gil Cebrian J, Díaz-Alersi R, Jesús Coma M, Gil Bello D. Principios de urgencia, emergencias y cuidados críticos. Capítulo 2.6 Patología pleural. http://umeet.united.edu/ tratado/c02607.htlm (Accedido el 15 de marzo 2005)

8.- González Montaner L J, González Montaner P J. Infecciones Pleuropulmonares. http://www.dic.org.ar/ enfpleu/epp078.php (Accedido el 15 de marzo 2005)

9.- Ingram R J, Call S, Andrade A, White C, Wheeler D. Management and outcome of pneumothorax in patients infected with human immunodeficiency virus. Clin Infect Dis 1996; 23: 624-7.

10.- Afessa B. Pleural effusion and pneumothorax in hospitalized patients with HIV infection. Chest. 2000; 117: 1031-7.

11.- Martínez-Vázquez C, Seijas M, Ocampo A, López A, Oliveira I, Sopena B, et al. Pneumothorax in patients infected by the human immunodeficiency virus. An Med Interna 2001; 18: 521-4.

12.- Beers M F, Sohn M, Swartz M. Recurrent pneumothorax in AIDS patients with Pneumocystis carinii pneumonia: a clinicopathologic report of three cases and review of the literature. Chest 1990; 98: 266-70.

13.- Benavides F, Medina B, Valeria C, Abuabuad R, De La Torre J M, Castillo M, et al. Neumotórax espontáneo primario en adultos. Rev Chil Cirugía 1999; 51: 134-9.

14.- Seguel E, Saldías R, Alarcón E, Gyhra A, González R, Bravo J, et al. Manejo del neumotórax espontáneo: Experiencia en 10 años. Rev Chil Cirugía 2004; 56:
61-5.

15.- Trachiotis G D, Vricella L A, Alyono D, Aaron B, Hix W. Management of AIDSrelated pneumothorax. Ann Thorac Surg 1996; 62: 1608-13.

16.- Vricella L A, Trachiotis G D. Heimlich valve in the manegement of pneumothorax in patients with advance AIDS. Chest 2001; 120: $15-8$.

17.- Hollander H, Mitchell K. Infección por VIH En Tierney L, McPhee M, Papadakis M. Diagnóstico clínico y tratamiento. $32^{\mathrm{a}}$ edición. México D. F. Editorial El Manual Moderno 1997; 1155-78.

18.- Horowitz M D, Oliva H. Pneumothorax in AIDS patients: operative management. Ann Surg 1993; 59: 200-4.

19.- Read C A, Reddy V D, Omara T E, Richardson M S. Doxycycline pleurodesis for pneumothorax in patients with AIDS. Chest 1994; 105: 823-5.

20.- Kimmel R D, Karp M P, Cascone J J, Zinns J S. Talc pleurodesis during videothoracoscopy for Pneumocystis carinii pneumonia-related pneumothorax: a new technique. Chest 1994; 105: 314-5.

21.- Aparicio R, Pereira J, Erskine P, Cid F. Sellamiento pleural terapéutico con fibrina. Rev Chil Enf Resp 1992; 8: 102-8.

22.- Suárez C, Czischke C, Lemus J, Santolaya R, Berrios R, Mena P, et al. Tratamiento videotoracoscópico del neumotórax espontáneo. Rev Chil Cirugía 1998; 50: 304-7. 